\title{
Analysis of mathematical model of leukemia
}

\author{
Mohamed Helal ${ }^{1, \mathrm{a}}$, Mostafa Adimy ${ }^{2, \mathrm{~b}}$, Abdelkader Lakmeche ${ }^{1, \mathrm{c}}$, and Laurent Pujo-Menjouet ${ }^{2, \mathrm{~d}}$ \\ ${ }^{1}$ Laboratory of Biomathematics, Univ. Sidi Bel Abbes, PB. 89, Sidi Bel Abbes 22000, Algeria \\ ${ }^{2}$ Université de Lyon, CNRS UMR 5208, Université Lyon 1, Institut Camille Jordan, 43 blvd. du \\ 11 novembre 1918, 69622 Villeurbanne Cedex, France
}

\begin{abstract}
In this paper, a model describing the dynamic of chronic myeloid leukemia is studied. By analyzing the corresponding characteristic equations, the local stability of trivial and nontrivial equilibria are discussed. By establishing appropriate Lyapunov functions, we prove the global stability of the positive constant equilibrium solutions.
\end{abstract}

\section{Introduction}

Several recent mathematical models have been developed to study the dynamics of chronic myeloid leukemia (CML) under IMATINIB treatment, see ([2, 3, 5, 6]). In all of these studies, the authors conclude that IMATINIB does not completely eliminate leukemic cells, and propose that IMATINIB therapy should be combined with an additional form of treatment.

In [5], the authors analyze a four-compartment differential equation model based on the known biology of the hematopoietic system to investigate the dynamics of CML with IMATINIB treatment. In their model, Michor et al. [5] study the interaction between leukemic cells and IMATINIB, they assume that these cells differentiate through four stages of their life cycle, beginning with leukemia stem cells. IMATINIB reduces the rate at which leukemic cells pass from one stage to the next, causing a rapid drop in the leukemia population. Based on their assumptions and analysis, they propose that leukemia inevitably persists, because IMATINIB hinders the differentiation of differentiated leukemic cells, but does not affect the leukemia stem cells. In particular, Michor et al. [5] hypothesizes that there is always a steadily growing population of leukemia stem cells despite IMATINIB treatment. As a result, based on their model, the leukemia population under IMATINIB eventually relapses, regardless of whether the model considers IMATINIB resistance mutations.

In [2], the authors incorporate the anti-leukemia immune response in CML patients on IMATINIB therapy to the model proposed in [5] by adding interactions with anti-leukemia T-cells. They formulate their mathematical model as a system of delay differential equations, and prove that the immune

\footnotetext{
a e-mail: mhelal_abbes@yahoo.fr

b e-mail: mostafa.adimy@inria.fr

${ }^{c}$ e-mail: lakmeche@yahoo.fr

d e-mail: pujo@math.univ-lyon1.fr
}

This is an Open Access article distributed under the terms of the Creative Commons Attribution License 4.0, which permits unrestricted use, distribution, and reproduction in any medium, provided the original work is properly cited. 
response may play a critical role in determining the length of time that CML patients under IMATINIB treatment remain in remission.

In [6], Roeder et al. develop a similar model of CML and IMATINIB. However, they subdivide the leukemia stem cells into two compartments: proliferating and quiescent cells. Proliferating leukemia stem cells are affected by IMATINIB, while quiescent leukemia stem cells are not affected. Due to this additional assumption, the leukemia population under IMATINIB does not relapse without the effects of IMATINIB resistance mutations. Instead, under IMATINIB treatment, the leukemia stem cell population restabilizes at lower equilibrium level and does not continue growing as in the Michor model.

Both [5] and [6] propose that IMATINIB does not eliminate the leukemia stem cell population. Consequently, the papers conclude that IMATINIB therapy should be combined with an additional treatment that either directly impacts leukemia stem cells or causes leukemia stem cells to become vulnerable to IMATINIB.

As an alternative approach, Komorova and Wodarz develop a model that focuses on the drug resistance of leukemia cells [3]. In their model, they implicitly assume that IMATINIB affects all leukemia cells including stem cells and that inevitable relapse is a result of acquired IMATINIB resistance mutations. Komorova and Wodarz consider the possibility of treating patients with multiple drugs to reduce the probability of any leukemic cell eventually acquiring resistance-mutations to all drugs. They determine that a treatment strategy consisting of three leukemia-targeted drugs of different specificity might have a strong chance of eliminating the disease.

The four approaches discussed above present a variety of hypotheses for the dynamics of IMATINIB treatment on leukemic cells. These papers also propose potential treatment strategies to enhance the effectiveness of IMATINIB. However, the difficulty with these treatments is that it is unclear what kind of drug could be used to target leukemia stem cells or what alternative drugs could be used in addition to IMATINIB for a multiple-drug strategy.

In this work, we consider the following more general mathematical model which is an extension of a model proposed in [5]. In our model, we assume that normal (resp. leukemic) cells differentiate through two stages of their life cycle, beginning with leukemic stem cells which produce produce progenitors.

The mathematical form of the system we shall investigate satisfies

$$
\begin{aligned}
& \dot{x}_{0}=\left(\beta-a_{x}-\beta_{0} x_{0}-\lambda\left(x_{1}+y_{1}+z_{1}\right)\right) x_{0}, \\
& \dot{x}_{1}=a_{x} x_{0}-d_{1} x_{1}, \\
& \dot{y}_{0}=\left(\gamma-a_{y}-\gamma_{0} y_{0}-\lambda\left(x_{1}+\alpha\left[y_{1}+z_{1}\right]\right)\right) y_{0}-r y_{0}, \\
& \dot{y}_{1}=a_{y} y_{0}-d_{2} y_{1}, \\
& \dot{z}_{0}=\left(\gamma-a_{z}-\gamma_{0} z_{0}-\lambda\left(x_{1}+\alpha\left[y_{1}+z_{1}\right]\right)\right) z_{0}+r y_{0}, \\
& \dot{z}_{1}=a_{z} z_{0}-d_{3} z_{1},
\end{aligned}
$$

with the following initial conditions

$$
\begin{aligned}
& x_{0}(0)=x_{0}^{0} \geq 0, \quad y_{0}(0)=y_{0}^{0} \geq 0, \quad z_{0}(0)=z_{0}^{0} \geq 0, \\
& x_{1}(0)=x_{1}^{0} \geq 0, \quad y_{1}(0)=y_{1}^{0} \geq 0, \quad z_{1}(0)=z_{1}^{0} \geq 0 .
\end{aligned}
$$

The population of interest is divided into three compartments coming from dictated by the epidemiological stages; normal cells, sensitive leukemic cells and resistant leukemic cells. We assume that normal (resp. leukemic) cells differentiate through two stages of their life cycle, beginning with leukemic stem cells which produce. Tables 1 and 2 list the definitions and symbols (populations and parameters) used in our model. This paper is organized as follows. In the next section, we state and prove general criterion for the existence positive solutions of system (1). In Sect. 3, we will study the global dynamics of (1) by constructing a suitable Lyapunov function and using LaSalle's invariance principle rather than by using the theory of competitive systems, as has been done in [6]. This will 


\section{WMLS 2014}

Table 1. Symbols and definitions of populations.

\begin{tabular}{c||l}
\hline symbol & definition \\
\hline$x_{0}$ & normal stem cells \\
$x_{1}$ & normal progenitor cells \\
$y_{0}$ & leukemic sensitive stem cells \\
$y_{1}$ & leukemic sensitive progenitor cells \\
$z_{0}$ & leukemic resistant stem cells \\
$z_{1}$ & leukemic resistant progenitor cells \\
\hline
\end{tabular}

Table 2. Symbols and definitions of parameters.

\begin{tabular}{c||l}
\hline parameter & explanation \\
\hline$\beta_{0}$ & death rate of the normal stem cells \\
$\gamma_{0}$ & death rate of leukemic stem cells \\
$\beta$ & division rate of normal stem cells \\
$\gamma$ & division rate leukemic stem cells \\
$\lambda$ & competitive parameter of the stem and progenitor cells \\
$a_{x}$ & production rate of the normal stem cells \\
$a_{y}$ & produce rate of the leukemic stem cells \\
$d_{1}$ & death rates of the normal progenitors cells \\
$d_{2}$ & death rates of the leukemic progenitors cells \\
$d_{3}(r)$ & death rates of the normal leukemic progenitors cells \\
$r$ & resistant parameter \\
$\alpha$ & $0<\alpha<1$ \\
\hline
\end{tabular}

enable us to obtain the global asymptotic stability of the equilibrium point under some hypotheses and by a simpler method. This will enable us to obtain the global asymptotic stability of the equilibrium point under some hypotheses and by a simpler method. In Sect. 4, we use numerical simulations of our model to discuss biological significance of our results and indicate possible extensions to the study of more comprehensive models. The model (1) becomes as follow

$$
\left\{\begin{array}{l}
\dot{x}_{0}=\left(\beta-a_{x}-\beta_{0} x_{0}-\lambda\left(x_{1}+y_{1}+z_{1}\right)\right) x_{0}, \\
\dot{x}_{1}=a_{x} x_{0}-d_{1} x_{1}, \\
\dot{y}_{0}=\left(\gamma-a_{y}-\gamma_{0} y_{0}-\lambda\left(x_{1}+\alpha y_{1}+\alpha z_{1}\right)\right) y_{0}-r y_{0}, \\
\dot{y}_{1}=a_{y} y_{0}-d_{2} y_{1} \\
\dot{z}_{0}=\left(\gamma-a_{y}-\gamma_{0} z_{0}-\lambda\left(x_{1}+\alpha y_{1}+\alpha z_{1}\right)\right) z_{0}+r y_{0}, \\
\dot{z}_{1}=a_{y} z_{0}-d_{3}(r) z_{1},
\end{array}\right.
$$

with the following initial conditions

$$
\begin{aligned}
& x_{0}(0)=x_{0}^{0} \geq 0, \quad y_{0}(0)=y_{0}^{0} \geq 0, \quad z_{0}(0)=z_{0}^{0} \geq 0, \\
& x_{1}(0)=x_{1}^{0} \geq 0, \quad y_{1}(0)=y_{1}^{0} \geq 0, \quad z_{1}(0)=z_{1}^{0} \geq 0 .
\end{aligned}
$$

Throughout this paper, we assume that conditions

$$
\begin{gathered}
a_{x}<a_{y}, \\
a_{y}+r<\gamma, \\
a_{x}<\beta<\gamma,
\end{gathered}
$$




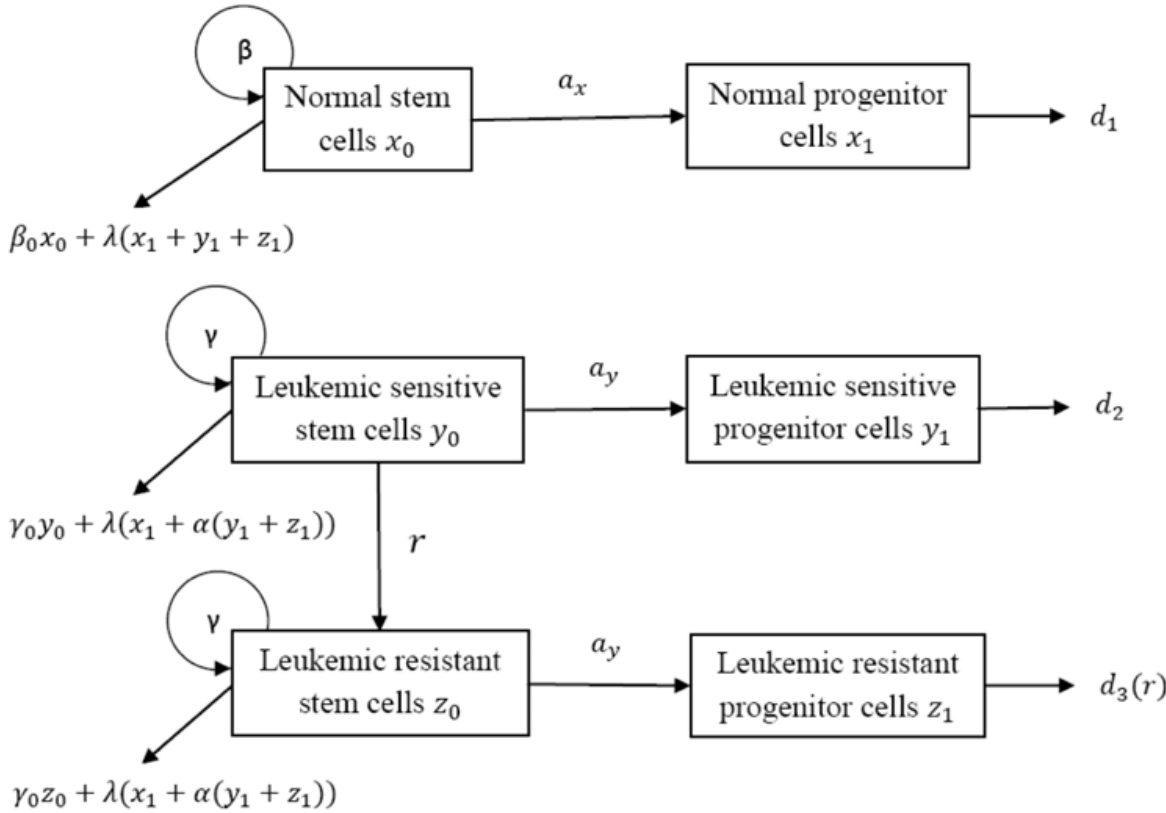

Figure 1. A state diagram for the model (3).

$$
0<\gamma_{0}<\beta_{0}
$$

and

$$
d_{3}(r) \leq d_{2}<d_{1} \text {, where } d_{3} \text { is decreasing on } r \text { with } d_{3}(0)=d_{2}
$$

hold in order that the state variables $x_{0}, x_{1}, y_{0}, y_{1}, z_{0}, z_{1}$ have a biological meanings. The compartmental diagram of the system (3) appears in Fig. 1.

\section{Global Wel-Posedness}

First we show that solutions of system (3) are positive and bounded.

Proposition 2.1: The system (3) with initial conditions (4) has unique bounded solution in $\mathbb{R}_{+}^{6}$ defined for all $t \geq 0$.

Proof: Let $F: \mathbb{R}^{6} \rightarrow \mathbb{R}^{6}$ be given by $F\left(\left(\begin{array}{c}x_{0} \\ x_{1}\end{array}\right),\left(\begin{array}{c}y_{0} \\ y_{1}\end{array}\right),\left(\begin{array}{c}z_{0} \\ z_{1}\end{array}\right)\right)=\left(\left(\begin{array}{c}F_{1} \\ F_{2}\end{array}\right),\left(\begin{array}{c}F_{3} \\ F_{4}\end{array}\right),\left(\begin{array}{c}F_{5} \\ F_{6}\end{array}\right)\right)$ where

$$
\left\{\begin{array}{l}
F_{1}=\left(\beta-a_{x}-\beta_{0} x_{0}-\lambda\left(x_{1}+y_{1}+z_{1}\right)\right) x_{0}, \\
F_{2}=a_{x} x_{0}-d_{1} x_{1} \\
F_{3}=\left(\gamma-a_{y}-\gamma_{0} y_{0}-\lambda\left(x_{1}+\alpha\left[y_{1}+z_{1}\right]\right)\right) y_{0}-r y_{0}, \\
F_{4}=a_{y} y_{0}-d_{2} y_{1} \\
F_{5}=\left(\gamma-a_{y}-\gamma_{0} z_{0}-\lambda\left(x_{1}+\alpha\left[y_{1}+z_{1}\right]\right)\right) z_{0}+r y_{0}, \\
F_{6}=a_{y} z_{0}-d_{3}(r) z_{1} .
\end{array}\right.
$$




\section{WMLS 2014}

Observe that $F$ is Lipschitz continuous on bounded sets of $\mathbb{R}^{6}$. Furthermore, for $t \geq 0$ and $\left(\left(\begin{array}{l}x_{0}(t) \\ x_{1}(t)\end{array}\right),\left(\begin{array}{l}y_{0}(t) \\ y_{1}(t)\end{array}\right),\left(\begin{array}{l}z_{0}(t) \\ z_{1}(t)\end{array}\right)\right) \in \mathbb{R}^{6}$ it follows that $F_{1} \geq 0$ for $x_{0}=0, F_{2} \geq 0$ for $x_{1}=0, F_{3} \geq 0$ for $y_{0}=0, F_{4} \geq 0$ for $y_{1}=0, F_{5} \geq 0$ for $z_{0}=0$ and $F_{6} \geq 0$ for $z_{1}=0$. Thus there exists a unique nonnegative solution to (3) in $\mathbb{R}_{+}^{6}$ for $t \geq 0$.

From the six equations of (3) we get $N=x_{0}+x_{1}+y_{0}+y_{1}+z_{0}+z_{1}$,

$$
\begin{aligned}
\dot{N} & \leq(\beta+1) x_{0}-x_{0}-d_{1} x_{1}+(\gamma+1) y_{0}-y_{0}-d_{2} y_{1}+(\gamma+1) z_{0}-z_{0}-d_{3}(r) z_{1} \\
& \leq(\beta+1) M_{x_{0}}+(\gamma+1)\left(M_{y_{0}}+M_{z_{0}}\right)-\rho N,
\end{aligned}
$$

where $M_{x_{0}}=\max \left(x_{0}^{0}, \frac{\beta-a_{x}}{\beta_{0}}\right), M_{y_{0}}=\max \left(y_{0}^{0}, \frac{\gamma-a_{y}-r}{\gamma_{0}}\right), M_{z_{0}}=\max \left(z_{0}^{0}, \frac{\gamma-a_{y}+\sqrt{\left(\gamma-a_{y}\right)^{2}+4 r \gamma_{0} M_{y_{0}}}}{2 \gamma_{0}}\right)$ and $\rho=\min \left(1, d_{3}(r)\right)=d_{3}(r)$. Hence we obtain the boundedness

$$
0 \leq N(t) \leq \frac{\rho}{(\beta+1) M_{x_{0}}+(\gamma+1)\left(M_{y_{0}}+M_{z_{0}}\right)}+N(0) e^{-\rho t} .
$$

It follows that $N(t) \leq \max \left(\frac{\rho}{(\beta+1) M_{x_{0}}+(\gamma+1)\left(M_{y_{0}}+M_{z_{0}}\right)}, N(0)\right)$, whenever $\left(\left(\begin{array}{c}x_{0}^{0} \\ x_{1}^{0}\end{array}\right),\left(\begin{array}{c}y_{0}^{0} \\ y_{1}^{0}\end{array}\right),\left(\begin{array}{c}z_{0}^{0} \\ z_{1}^{0}\end{array}\right)\right) \in \mathbb{R}_{+}^{6}$ and $t \geq 0$, this implies boundedness of solutions.

Thus the existence of a unique global nonnegative bounded solution is proved.

\section{Case $r=0$}

In this section, we consider the case where the resistant parameter $r=0$ with $z_{0}=z_{1}=0$.

We obtain the following system

$$
\left\{\begin{array}{l}
\dot{x}_{0}=\left(\beta-a_{x}-\beta_{0} x_{0}-\lambda\left(x_{1}+y_{1}\right)\right) x_{0}, \\
\dot{x}_{1}=a_{x} x_{0}-d_{1} x_{1}, \\
\dot{y}_{0}=\left(\gamma-a_{y}-\gamma_{0} y_{0}-\lambda\left(x_{1}+\alpha y_{1}\right)\right) y_{0}, \\
\dot{y}_{1}=a_{y} y_{0}-d_{2} y_{1} .
\end{array}\right.
$$

\subsection{Steady states analysis}

Proposition 3.1: Let $\lambda \geq 0$ and assume that the conditions (5)-(9) are satisfied. Then the system (10) has three trivial equilibrium, $E_{0}=\left(\left(\begin{array}{l}0 \\ 0\end{array}\right),\left(\begin{array}{l}0 \\ 0\end{array}\right)\right)$ which symbolizes extinction of a population cells, $E_{1}=\left(\left(\begin{array}{c}\xi_{1} \\ \frac{a_{x}}{d_{1}} \xi_{1}\end{array}\right),\left(\begin{array}{l}0 \\ 0\end{array}\right)\right)$ namely the disease free equilibrium, and $E_{2}=\left(\left(\begin{array}{l}0 \\ 0\end{array}\right),\left(\begin{array}{c}\eta_{2} \\ \frac{a_{y}}{d_{2}} \eta_{2}\end{array}\right)\right)$ which we call the healthy free equilibrium.

In addition, for $d_{1}>\lambda \frac{a_{x}}{\beta_{0}}\left(\frac{\beta-a_{x}}{\gamma-a_{y}}-1\right)$ and $d_{2}>\lambda \frac{a_{y}}{\gamma_{0}} \frac{\gamma-a_{y}}{\beta-a_{x}}-\alpha$, system (10) admits another nontrivial equilibrium $E_{3}=\left(\left(\begin{array}{c}\xi_{3} \\ \frac{a_{x}}{d_{1}} \xi_{3}\end{array}\right),\left(\begin{array}{c}\eta_{3} \\ \frac{a_{y}}{d_{2}} \eta_{3}\end{array}\right)\right)$ which we call the endemic equilibrium. The expression of $\xi_{i}$ and $\eta_{i}$ are given in Table 3 .

Remark 3.2: For $\alpha<\frac{\gamma-a_{y}}{\beta-a_{x}}<1, d_{2}=\lambda \frac{a_{y}}{\gamma_{0}}\left(\frac{\gamma-a_{y}}{\beta-a_{x}}-\alpha\right)$ and $d_{1}=\lambda \frac{a_{x}}{\beta_{0}}\left(\frac{\beta-a_{x}}{\gamma-a_{y}}-1\right)$ we have an infinity of equilibria of the form $E_{3}$. 
Table 3. Equilibrium formulation.

\begin{tabular}{l||l|l}
\hline equilibrium & name & expression \\
\hline$E_{1}=\left(\left(\begin{array}{c}\xi_{1} \\
\frac{a_{x}}{d_{1}} \xi_{1}\end{array}\right),\left(\begin{array}{l}0 \\
0\end{array}\right)\right)$ & disease free equilibrium & $\xi_{1}=\frac{\left(\beta-a_{x}\right) d_{1}}{\beta_{0} d_{1}+\lambda a_{x}}$ \\
\hline$E_{2}=\left(\left(\begin{array}{l}0 \\
0\end{array}\right),\left(\begin{array}{c}\eta_{2} \\
\frac{a_{y}}{d_{2}} \eta_{2}\end{array}\right)\right)$ & healthy free equilibrium & $\eta_{2}=\frac{\left(\gamma-a_{y}\right) d_{2}}{\gamma_{0} d_{2}+\lambda \alpha a_{y}}$ \\
\hline$E_{3}=\left(\left(\begin{array}{c}\xi_{3} \\
\frac{a_{x}}{d_{1}} \xi_{3}\end{array}\right),\left(\begin{array}{c}\eta_{3} \\
\frac{a_{y}}{d_{2}} \eta_{3}\end{array}\right)\right)$ & endemic equilibrium & $\xi_{3}=\frac{\xi_{1}-\frac{\lambda a_{y} d_{1}}{\left(\beta_{0} d_{1}+\lambda a_{x}\right) d_{2}} \eta_{2}}{1-\frac{\lambda a_{x} \lambda a_{y}}{\left(\gamma_{0} d_{2}+\lambda \alpha a_{y}\right)\left(\beta_{0} d_{1}+\lambda a_{x}\right)}}$ \\
& $\eta_{3}=\frac{\lambda a_{x} d_{2}}{1-\frac{\lambda a_{x} \lambda a_{y}}{\left(\gamma_{0} d_{2}+\lambda \alpha a_{y}\right) d_{1}} \xi_{1}}$ \\
\hline
\end{tabular}

\subsection{Stability analysis and bifurcation}

Let $q=\frac{\gamma-a_{y}}{\beta-a_{x}}, q_{1}=\frac{4 \gamma_{0}}{\beta_{0}+4 \gamma_{0}}, q_{2}=\frac{\gamma_{0}+4 \alpha^{2} \beta_{0}}{4 \alpha \beta_{0}}, d_{1}^{*}=\frac{\lambda a_{x}}{\beta_{0}}\left(\frac{1-q}{q}\right), d_{1}^{\bullet}=\frac{1}{4} \frac{\lambda a_{x}}{\gamma_{0}}, d_{2}^{*}=\frac{\lambda a_{y}}{\gamma_{0}}(q-\alpha)$ and $d_{2}^{\bullet}=\frac{1}{4 \alpha} \frac{\lambda a_{y}}{\beta_{0}}$.

\subsubsection{Local asymptotic stability}

To analyze the stability of these steady states we compute their linearizations.

At the point $E_{i}=\left(\left(\begin{array}{c}\xi_{i} \\ \frac{a_{x}}{d_{1}} \xi_{i}\end{array}\right),\left(\begin{array}{c}\eta_{i} \\ \frac{a_{y}}{d_{2}} \eta_{i}\end{array}\right)\right)$, we obtain the linearization

$$
A=\left(\begin{array}{cccc}
\lambda_{1} & -\lambda \xi_{i} & 0 & -\lambda \xi_{i} \\
a_{x} & -d_{1} & 0 & 0 \\
0 & -\lambda \eta_{i} & \lambda_{2} & -\lambda \alpha \eta_{i} \\
0 & 0 & a_{y} & -d_{2}
\end{array}\right)
$$

where

$\lambda_{1}=\beta-a_{x}-\lambda\left(\frac{a_{x}}{d_{1}} \xi_{i}+\frac{a_{y}}{d_{2}} \eta_{i}\right)-2 \beta_{0} \xi_{i}$, and

$\lambda_{2}=\gamma-a_{y}-\lambda\left(\frac{a_{x}}{d_{1}} \xi_{i}+\alpha \frac{a_{y}}{d_{2}} \eta_{i}\right)-2 \gamma_{0} \eta_{i}$.

Theorem 3.3: Let $\lambda \geq 0$, and assume that the conditions (5)-(9) are satisfied. Then

1. $E_{0}$ is unstable.

2. If $q<1$ and $d_{1}<d_{1}^{*}$, then $E_{1}$ is locally asymptotically stable. If either $q>1$ or $d_{1}>d_{1}^{*}$, then $E_{1}$ is unstable.

3. If $q>\alpha, d_{2}<d_{2}^{*}$, then $E_{2}$ is locally asymptotically stable. If either $q<\alpha$ or $d_{2}>d_{2}^{*}$, then $E_{2}$ is unstable.

4. If $d_{1}>d_{1}^{*}$ and $d_{2}>d_{2}^{*}$, then $E_{3}$ is locally asymptotically stable.

Proof: From the linearization (11), we obtain

1. At point $E_{0}$, the values $\beta-a_{x}>0,-d_{1}, \gamma-a_{y}>0$ and $-d_{2}$ are the eigenvalues of $A$. We conclude that the trivial equilibrium $E_{0}$ is unstable. 
2. At point $E_{1}$ we get $\lambda_{1}=-\beta_{0} \xi_{1}$ and $\lambda_{2}=\gamma-a_{y}-\lambda \frac{a_{x}}{d_{1}} \xi_{1}$. The eigenvalues of $A$ are $\lambda_{2},-d_{2}$ and the roots of the characteristic polynomial of the minor matrix of $A$ given by

$$
P(u)=\left(\lambda_{1}-u\right)\left(-d_{1}-u\right)+\lambda a_{x} \xi_{1}=0 .
$$

This equation is equivalent to

$$
P(u)=u^{2}+e_{1} u+e_{2}=0,
$$

where $e_{1}=d_{1}-\lambda_{1}$ and $e_{2}=-d_{1} \lambda_{1}+\lambda a_{x} \xi_{1}$.

Since $e_{1}>0$ and $e_{2}>0$, the Ruth-Hurwitz criterion implies that all roots of $P$ have negative real parts. Hence eigenvalues of $A$ have negative real parts if $\lambda_{2}<0$, which shows that the equilibrium $E_{1}$ is locally asymptotically stable if $q<1$ and $d_{1}<d_{1}^{*}$.

3. At point $E_{2}$ we have $\lambda_{1}=\beta-a_{x}-\lambda \frac{a_{y}}{d_{2}} \eta_{2}$ and $\lambda_{2}=-\gamma_{0} \eta_{2}$. The eigenvalues of $A$ are $\lambda_{1},-d_{1}$ and the roots of the characteristic polynomial of the minor matrix of $A$ given by

$$
P(u)=\left(\lambda_{2}-u\right)\left(-d_{2}-u\right)+\lambda \alpha a_{y} \eta_{2}=0 .
$$

This equation is equivalent to

$$
P(u)=u^{2}+e_{1} u+e_{2},
$$

where $e_{1}=d_{2}-\lambda_{2}$ and $e_{2}=-d_{2} \lambda_{2}+\lambda \alpha a_{y} \eta_{2}$.

Since $e_{1}>0$ and $e_{2}>0$, the Ruth-Hurwitz criterion implies that all roots of $P$ have negative real parts. Hence eigenvalues of $A$ have negative real parts if $\lambda_{1}<0$, which shows that the equilibrium $E_{2}$ is locally asymptotically stable if $q>\alpha, d_{2}<d_{2}^{*}$.

4. At point $E_{3}$ we have $\lambda_{1}=-\beta_{0} \xi_{3}$ and $\lambda_{2}=-\gamma_{0} \eta_{3}$. The eigenvalues of $A$ are the roots of the characteristic polynomial of the minor matrix of $A$ given by

$$
P(u)=\left[\left(\lambda_{1}-u\right)\left(-d_{1}-u\right)+\lambda a_{x} \xi_{3}\right]\left[\left(\lambda_{2}-u\right)\left(-d_{2}-u\right)+\lambda \alpha a_{y} \eta_{3}\right]-a_{x} a_{y} \lambda^{2} \xi_{3} \eta_{3}=0 .
$$

This equation is equivalent to

$$
u^{4}+e_{1} u^{3}+e_{2} u^{2}+e_{3} u+e_{4}=0
$$

where

$$
\begin{aligned}
& e_{1}=d_{1}+\beta_{0} \xi_{3}+d_{2}+\gamma_{0} \eta_{3}>0, \\
& e_{2}=\left(\beta_{0} d_{1}+\lambda a_{x}\right) \xi_{3}+\left(\gamma_{0} d_{2}+\lambda \alpha a_{y}\right) \eta_{3}+\left(d_{1}+\beta_{0} \xi_{3}\right)\left(d_{2}+\gamma_{0} \eta_{3}\right)>0, \\
& e_{3}=\left(d_{1}+\beta_{0} \xi_{3}\right)\left(\gamma_{0} d_{2}+\lambda \alpha a_{y}\right) \eta_{3}+\left(d_{2}+\gamma_{0} \eta_{3}\right)\left(\beta_{0} d_{1}+\lambda a_{x}\right) \xi_{3}>0 \text { and } \\
& e_{4}=\left[\left(\beta_{0} d_{1}+\lambda a_{x}\right)\left(\gamma_{0} d_{2}+\lambda \alpha a_{y}\right)-a_{x} a_{y} \lambda^{2}\right] \xi_{3} \eta_{3}>0 \text { since } d_{2}>d_{2}^{*} \text {. Moreover, } e_{1} e_{2} e_{3}>e_{3}^{2}+ \\
& e_{1}^{2} e_{4}, \text { for the Routh-Hurwitz criterion, it follows that all roots of } A \text { have negative real parts if } \\
& d_{2}>d_{2}^{*} \text {, which shows that the equilibrium } E_{3} \text { is locally asymptotically stable if } d_{1}>d_{1}^{*} \text { and } \\
& d_{2}>d_{2}^{*} .
\end{aligned}
$$

In summary we have

Table 4. Summary of the model with $r=0$.

\begin{tabular}{|l|l||l|l||l|l|}
\hline \multicolumn{3}{|c||}{ Case 1: $q \leq \alpha$} & \multicolumn{2}{c||}{ Case $1: \alpha<q<1$} & \multicolumn{2}{c|}{ Case 3: $1 \leq q$} \\
\hline region & stability & region & stability & region & stability \\
\hline I & $E_{1}$ is L.A.S. & I & $E_{1}$ is L.A.S. & I disappear & \\
& $E_{2}$ is unstable & & $E_{2}$ is unstable & & \\
\hline II disappear & & II & $E_{1}$ is unstable & II & $E_{1}$ is unstable \\
& & & $E_{2}$ is L.A.S. & & $E_{2}$ is L.A.S. \\
\hline III & $E_{1}$ is unstable & III & $E_{1}$ is unstable & III & $E_{1}$ is unstable \\
& $E_{2}$ is unstable & & $E_{2}$ is unstable & & $E_{2}$ is unstable \\
& $E_{3}$ is L.A.S. & & $E_{3}$ is L.A.S. & & E \\
& & IV & $E_{1}$ is L.A.S. & IV disappear & \\
\hline IV disappear & & & $E_{2}$ is L.A.S. & & \\
& & & & &
\end{tabular}


ITM Web of Conferences

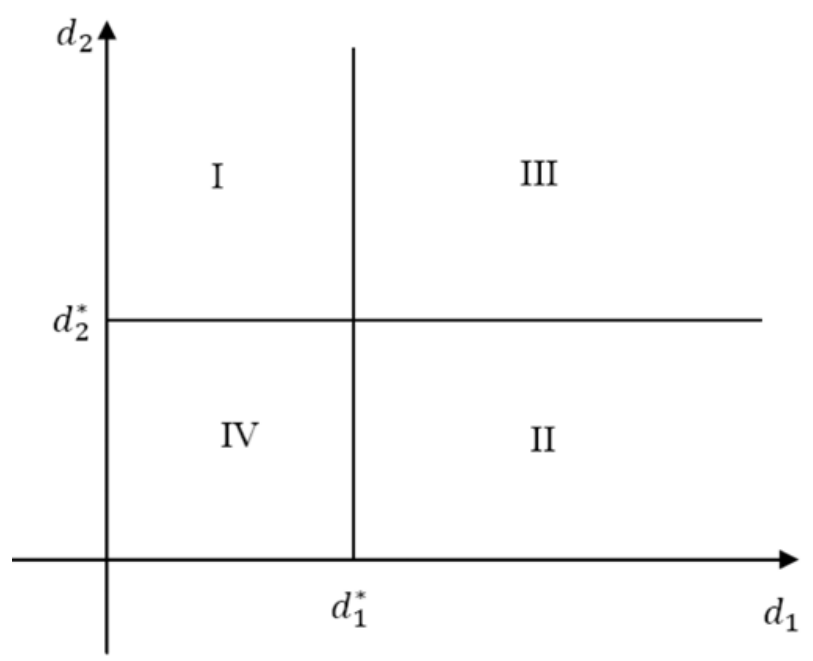

Figure 2. Bifurcation diagram for the model when $\alpha<q<1$. In region I, the disease free equilibrium $E_{1}$ is locally asymptotically stable, in region II, the healthy free equilibrium $E_{2}$ is locally asymptotically stable, in region III, the endemic equilibrium $E_{3}$ is locally asymptotically stable and region IV represents the local asymptotic stability of disease free equilibrium $E_{1}$ and healthy free equilibrium $E_{2}$.

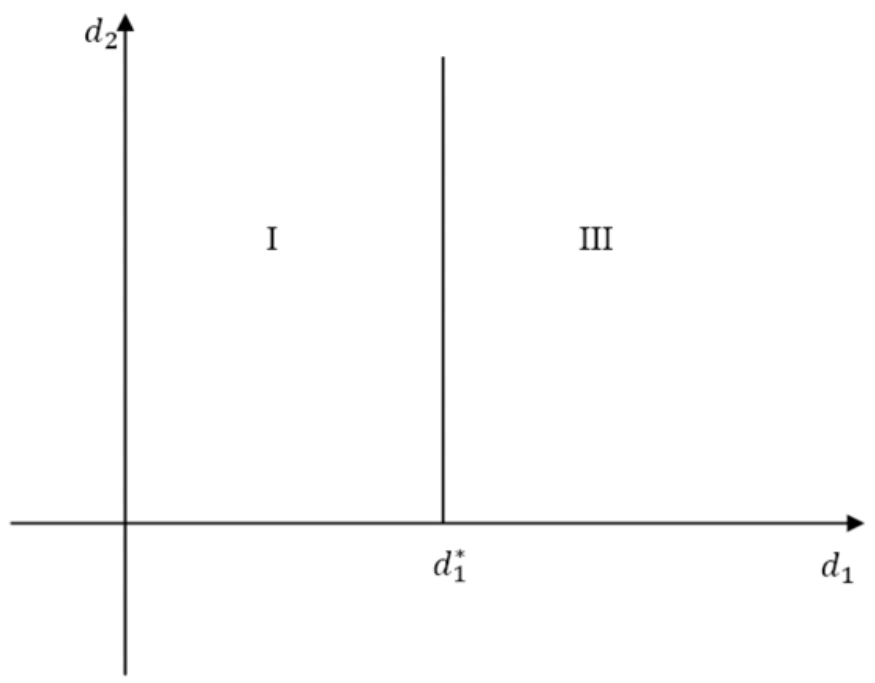

Figure 3. Bifurcation diagram for the model when $q \leq \alpha$. The regions II and IV disappear.

where

I: $d_{1}<d_{1}^{*}$ and $d_{2}>d_{2}^{*}$,

II: $d_{1}>d_{1}^{*}$ and $d_{2}<d_{2}^{*}$,

III: $d_{1}>d_{1}^{*}$ and $d_{2}>d_{2}^{*}$, and

IV: $d_{1}<d_{1}^{*}$ and $d_{2}<d_{2}^{*}$.

Figures 2, 3, 4, 5 and 6 show the bifurcation diagram for this model. 
WMLS 2014

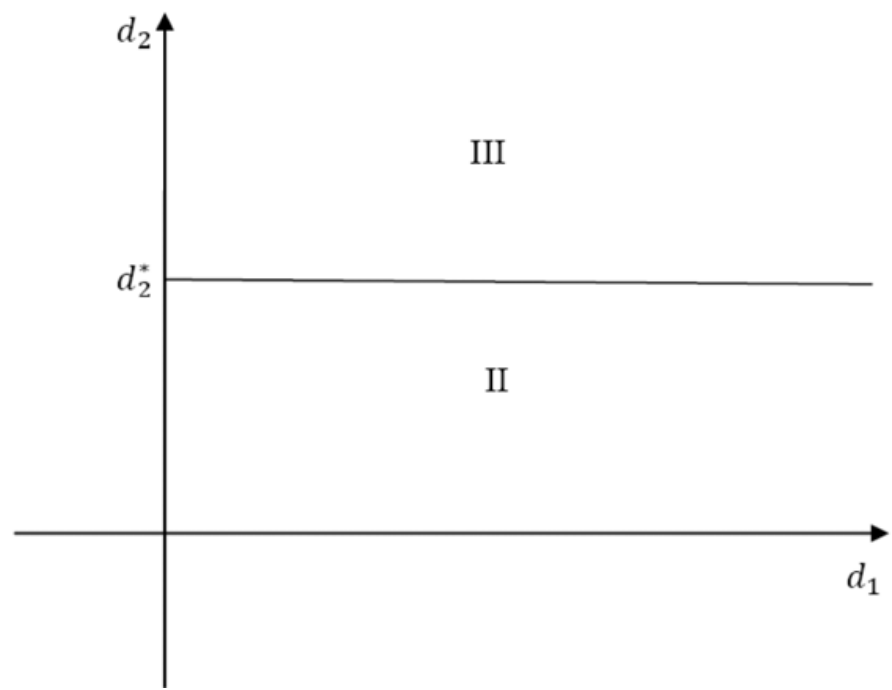

Figure 4. Bifurcation diagram for the model when $q>1$. The regions I and IV disappear.

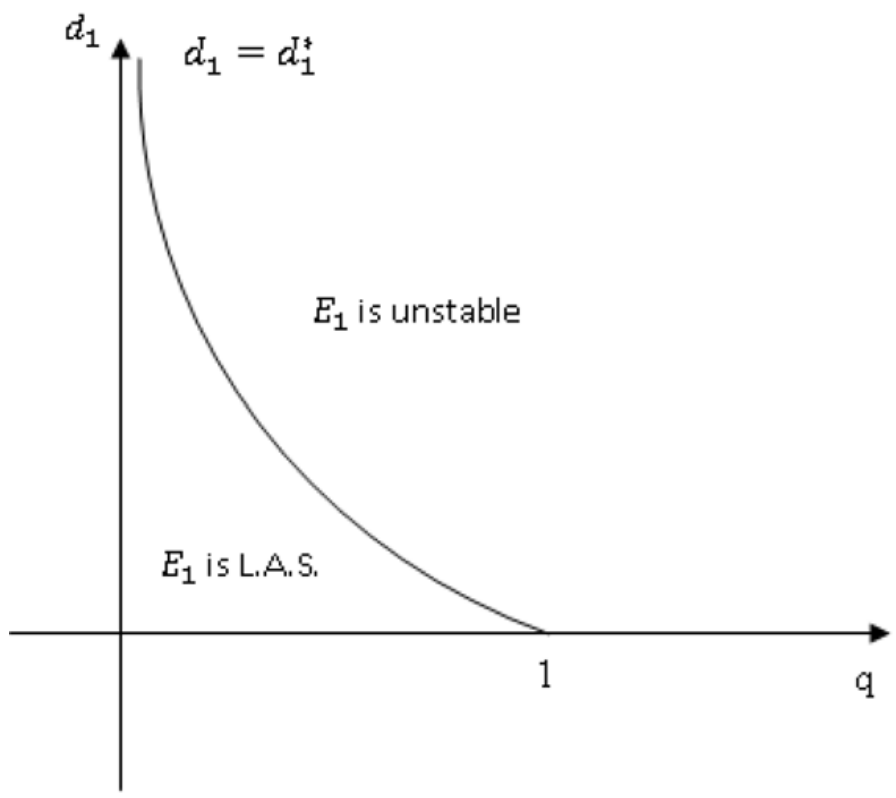

Figure 5. Bifurcation diagram for the DFE.

\subsubsection{Concept of $\mathcal{R}_{0}$}

The disease free equilibrium (DFE) for this nondimensionalized general model of chronic myeloid leukemia may be used to find the basic reproduction number $\mathcal{R}_{0}$, which indicates the average number of new infections. The basic epidemiological reproductive number is given by

$$
\mathcal{R}_{0}=\frac{\gamma}{\frac{\left(\beta-a_{x}\right) \lambda a_{x}}{\beta_{0} d_{1}+\lambda a_{x}}+a_{y}} .
$$

However, this nondimensional number is not enough to characterize the dynamics of model (4), (10). 


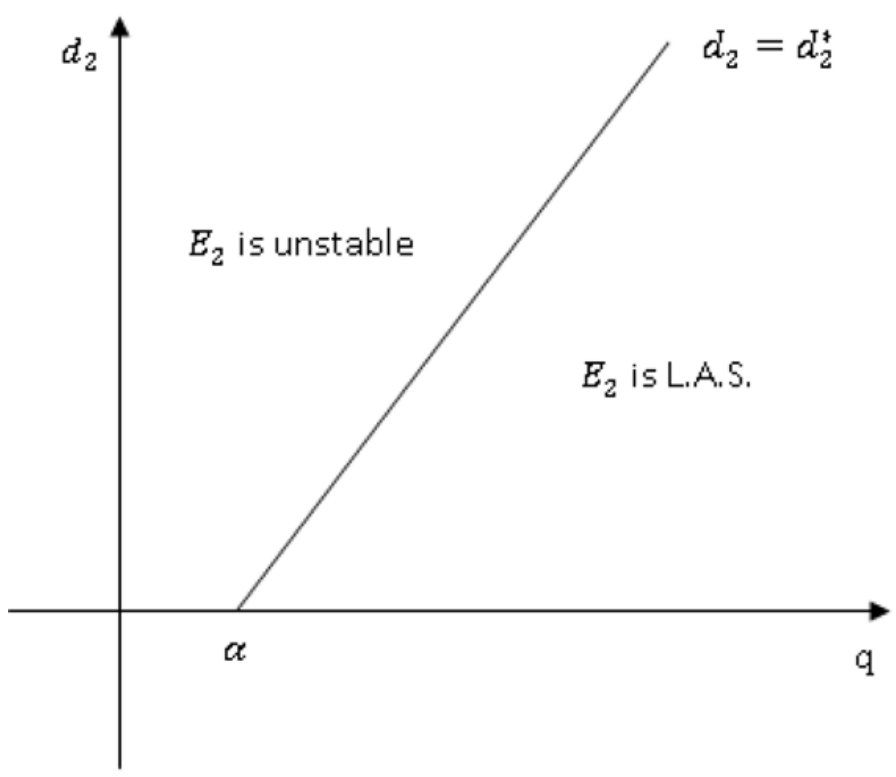

Figure 6. Bifurcation diagram for the HFE.

\subsubsection{Analysis at $\mathcal{R}_{0}=1$}

To analyze the local stability in critical cases, we use the center manifold theory, as described in CastilloChavez and Song (Theorem 4.1) [1]. Center manifold theory has been used to decide the local stability of a nonhyperbolic equilibrium (linearization matrix has at least one eigenvalue with zero real part). This theory can not only determine the local stability of the nonhyperbolic equilibrium but also settles the question of the existence of another equilibrium (bifurcated from the nonhyperbolic equilibrium).

To apply this method, the system (10) becomes

$$
\dot{x}=f(x, \phi)=\left\{\begin{array}{l}
\dot{x}_{0}(t)=\left(\beta-a_{x}-\beta_{0} x_{0}-\lambda\left(x_{1}+y_{1}\right)\right) x_{0}:=f_{1}, \\
\dot{x}_{1}(t)=a_{x} x_{0}-d_{1} x_{1}:=f_{2}, \\
\dot{y}_{0}(t)=\left(\gamma-a_{y}-\gamma_{0} y_{0}-\lambda\left(x_{1}+\alpha y_{1}\right)\right) y_{0}:=f_{3}, \\
\dot{y}_{1}(t)=a_{y} y_{0}-d_{2} y_{1}:=f_{4},
\end{array}\right.
$$

where $x=\left(\left(\begin{array}{l}x_{0} \\ x_{1}\end{array}\right),\left(\begin{array}{l}y_{0} \\ y_{1}\end{array}\right)\right), f=\left(f_{1}, f_{2}, f_{3}, f_{4}\right)$ and $\phi=\gamma-a_{y}$.

At $\mathcal{R}_{0}=1$, we obtain $\phi=\gamma-a_{y}=\frac{\left(\beta-a_{x}\right) \lambda a_{x}}{\beta_{0} d_{1}+\lambda a_{x}}$. From the linearization matrix of system (13) around the disease free equilibrium $E_{1}$ when $\mathcal{R}_{0}=1$, we can show that zero is a simple eigenvalue and all other eigenvalues have negative real parts. A right eigenvector $w$ corresponding to the zero eigenvalue is $w=\left(\left(\begin{array}{c}-\frac{\lambda a_{y} d_{1}}{\left(\beta_{0} d_{1}+\lambda a_{x}\right) d_{2}} \\ -\frac{\lambda a_{y} a_{x}}{\left(\beta_{0} d_{1}+\lambda a_{x}\right) d_{2}}\end{array}\right),\left(\begin{array}{c}1 \\ \frac{a_{y}}{d_{2}}\end{array}\right)\right)$, and the left eigenvector satisfying $v \cdot w=1$ is $v=\left(\left(\begin{array}{l}0 \\ 0\end{array}\right),\left(\begin{array}{l}1 \\ 0\end{array}\right)\right)$.

For system (13) the second partial derivatives of $f_{3}$ are given by

$$
\frac{\partial^{2} f_{3}}{\partial y_{0}^{2}}=-2 \gamma_{0}, \quad \frac{\partial^{2} f_{3}}{\partial y_{0} \partial x_{1}}=-\lambda, \quad \frac{\partial^{2} f_{3}}{\partial y_{0} \partial y_{1}}=-\lambda \alpha, \quad \frac{\partial^{2} f_{3}}{\partial y_{0} \partial z_{1}}=-\lambda \alpha, \quad \text { and } \quad \frac{\partial^{2} f_{3}}{\partial \phi \partial y_{0}}=1 .
$$




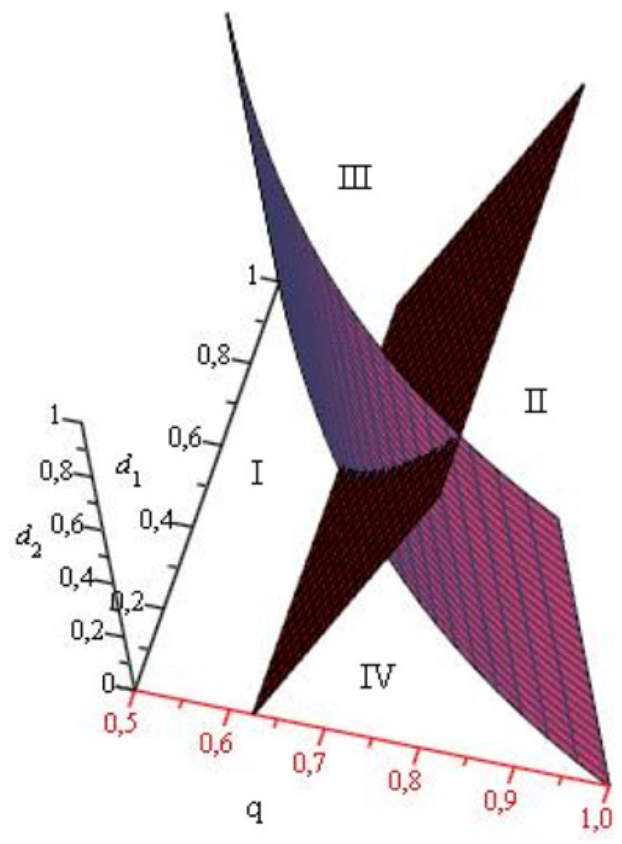

Figure 7. Bifurcation diagram for the model.
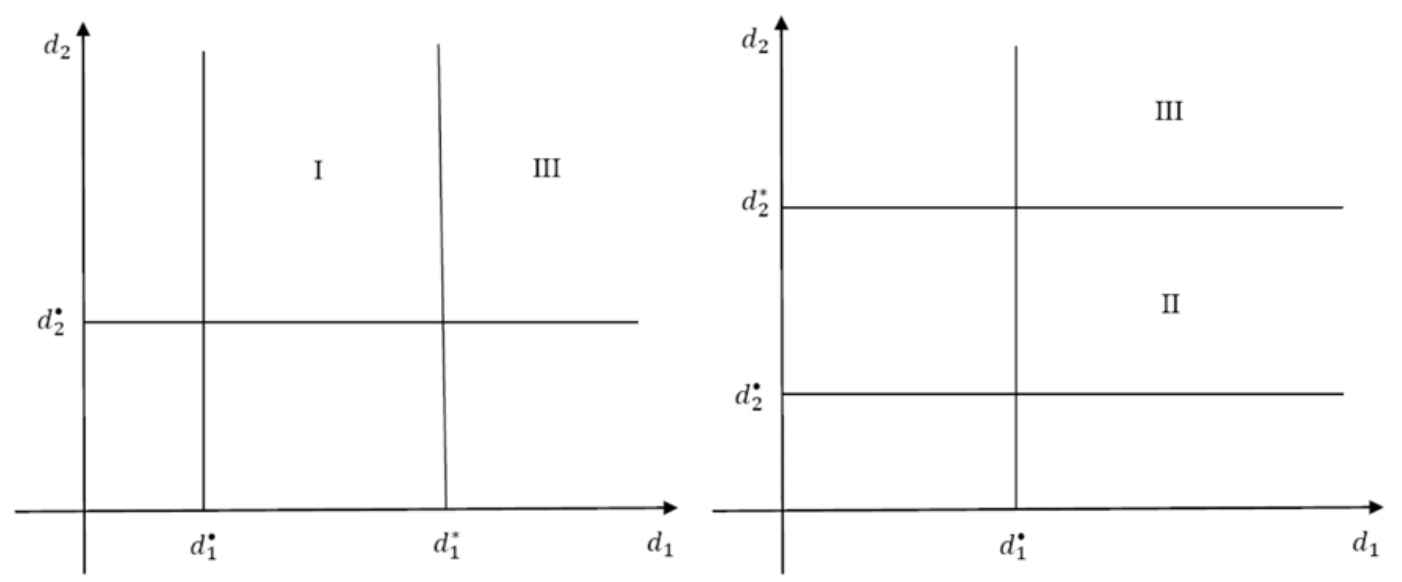

Figure 8. Bifurcation diagram for the model when $q<q_{1}$ (Resp. $q>q_{2}$ ). In region I, the disease free equilibrium $E_{1}$ is globally asymptotically stable, in region III, the endemic equilibrium $E_{3}$ is globally asymptotically stable (Resp. In region II, the healthy free equilibrium $E_{2}$ is globally asymptotically stable, in region III, the endemic equilibrium $E_{3}$ is globally asymptotically stable).

It follows that

$$
\begin{aligned}
a & =-2 v_{3} w_{3}\left(\lambda w_{2}+\gamma_{0} w_{3}+\lambda \alpha w_{4}\right) \\
& =-2\left(\lambda \frac{a_{y}}{d_{2}}\left(\alpha-\frac{\lambda a_{x}}{\beta_{0} d_{1}+\lambda a_{x}}\right)+\gamma_{0}\right)
\end{aligned}
$$


and

$$
b=v_{3} w_{3}=1>0 .
$$

Thus, from Theorem 4.1, item (iv) in Castillo-Chavez and Song [1], we have the following result.

Theorem 3.4: Let $d_{2}^{\prime}=\frac{\lambda a_{y}}{\gamma_{0}}\left(\frac{\lambda a_{x}}{\beta_{0} d_{1}+\lambda a_{x}}-\alpha\right)$ and $d_{1}^{\prime}=\frac{\lambda a_{x}}{\beta_{0}}\left(\frac{1-\alpha}{\alpha}\right)$.

1. If $d_{2}^{\prime}<d_{2}<d_{2}^{*}$, the unique endemic equilibrium disappears whenever $\mathcal{R}_{0}>1$ and is close to 1 .

2. If $d_{2}>d_{2}^{*}>d_{2}^{\prime}$, the unique endemic equilibrium is locally asymptotically stable whenever $\mathcal{R}_{0}>1$ and is close to 1 .

3. If $d_{2}<d_{2}^{\prime}$ and $d_{1}<d_{1}^{\prime}$, the unique endemic equilibrium disappears whenever $\mathcal{R}_{0}>1$ and is close to 1 .

\subsubsection{Global stability}

Let $T=\left(\left(\begin{array}{c}x_{0}-\xi_{i} \\ x_{1}-\frac{a_{x}}{d_{1}} \xi_{i}\end{array}\right),\left(\begin{array}{c}y_{0}-\eta_{i} \\ y_{1}-\frac{a_{y}}{d_{2}} \eta_{i}\end{array}\right)\right)$ and $A=\left(\begin{array}{cccc}\beta_{0} & 0 & 0 & \frac{\lambda}{2} \\ 0 & \frac{\lambda d_{1}}{a_{x}} & \frac{\lambda}{2} & 0 \\ 0 & \frac{\lambda}{2} & \gamma_{0} & 0 \\ \frac{\lambda}{2} & 0 & 0 & \frac{\lambda \alpha d_{2}}{a_{y}}\end{array}\right)$.

Next, we study the global stability of the steady states. The proof relies on the construction of a global Lyapunov functions.

\section{Theorem 3.5:}

1. If $q<q_{1}, d_{1}^{\bullet}<d_{1} \leq d_{1}^{*}$ and $d_{2}>d_{2}^{\bullet}$, then $E_{1}$ is globally asymptotically stable in $\mathbb{R}_{+}^{4} /\{0\} \times \mathbb{R}_{+}^{3}$.

2. If $q>q_{2}, d_{1}>d_{1}^{\bullet}$ and $d_{2}^{\bullet}<d_{2} \leq d_{2}^{*}$, then $E_{2}$ is globally asymptotically stable in $\mathbb{R}_{+}^{4} / \mathbb{R}_{+}^{2} \times$ $\{0\} \times \mathbb{R}_{+}$.

3. If $d_{1}>\max \left(d_{1}^{*}, d_{1}^{\bullet}\right)$ and $d_{2}>\max \left(d_{2}^{*}, d_{2}^{\bullet}\right)$, then $E_{3}$ is globally asymptotically stable in $\mathbb{R}_{+}^{4} /\{0\} \times \mathbb{R}_{+}^{3} \cup \mathbb{R}_{+}^{2} \times\{0\} \times \mathbb{R}_{+}$.

Proof:

1. Let us consider the Lyapunov function

$$
V_{1}=\left(x_{0}-\xi_{1}-\xi_{1} \ln \frac{x_{0}}{\xi_{1}}\right)+\frac{\lambda}{2 a_{x}}\left(x_{1}-\frac{a_{x}}{d_{1}} \xi_{1}\right)^{2}+y_{0}+\frac{\lambda \alpha}{2 a_{y}} y_{1}^{2} .
$$

It is easily seen that $V_{1} \geq 0$ and $V_{1}=0$ if and only if $x_{0}=\xi_{1}, x_{1}=\frac{a_{x}}{d_{1}} \xi_{1}$ and $y_{0}=y_{1}=0$. Calculating the time derivative of $V_{1}$ along the positive solutions of model (10), we obtain

$$
\begin{aligned}
\dot{V}_{1}= & -\beta_{0}\left(x_{0}-\xi_{1}\right)^{2}-\lambda\left(x_{0}-\xi_{1}\right) y_{1}-\frac{\lambda d_{1}}{a_{x}}\left(x_{1}-\frac{a_{x}}{d_{1}} \xi_{1}\right)^{2} \\
& +\left(\gamma-a_{y}-\lambda \frac{a_{x}}{d_{1}} \xi_{1}\right) y_{0}-\gamma_{0} y_{0}^{2}-\lambda\left(x_{1}-\frac{a_{x}}{d_{1}} \xi_{1}\right)+y_{0}-\frac{\lambda \alpha d_{2}}{a_{y}} y_{1}^{2} \\
= & -T A T^{\prime}+\left(\gamma-a_{y}-\lambda \frac{a_{x}}{d_{1}} \xi_{1}\right) y_{0} .
\end{aligned}
$$


The matrix $A$ is positive definite if $d_{1}>\frac{1}{4} \frac{\lambda a_{x}}{\gamma_{0}}$ and $d_{2}>\frac{1}{4 \alpha} \frac{\lambda a_{y}}{\beta_{0}}$. It follows from $q<1$ and $d_{1} \leq d_{1}^{*}$ that $\dot{V}_{1} \leq 0$ for all $x_{0}, x_{1}, y_{0}, y_{1}$ and $\dot{V}_{1}=0$, when $x_{0}=\xi_{1}, x_{1}=\frac{a_{x}}{d_{1}} \xi_{1}$ and $y_{0}=y_{1}=0$. Hence La Salle's theorem [4] implies convergence of the solutions to this equilibrium, for all initial values not in the set $\{0\} \times \mathbb{R}_{+}^{3}$. This shows that the disease free equilibrium $E_{1}$ is globally asymptotically stable in $\mathbb{R}_{+}^{4} /\{0\} \times \mathbb{R}_{+}^{3}$. If the initial data starts from $\{0\} \times \mathbb{R}_{+}^{3}$, then the solution obviously converges to the equilibria $E_{0}$ or $E_{2}$.

2. Let us consider the Lyapunov function

$$
V_{2}=x_{0}+\frac{\lambda}{2 a_{x}} x_{1}^{2}+\left(y_{0}-\eta_{2}-\eta_{2} \ln \frac{y_{0}}{\eta_{2}}\right)+\frac{\lambda \alpha}{2 a_{y}}\left(y_{1}-\frac{a_{y}}{d_{2}} \eta_{2}\right)^{2} .
$$

It is easily seen that $V_{2} \geq 0$ and $V_{2}=0$ if and only if $x_{0}=x_{1}=0, y_{0}=\eta_{2}$ and $y_{1}=\frac{a_{y}}{d_{2}} \eta_{2}$. Calculating the time derivative of $V_{2}$ along the positive solutions of model (10), we obtain

$$
\begin{aligned}
\dot{V}_{2}= & \left(\beta-a_{x}-\lambda \frac{a_{y}}{d_{3}} \eta_{2}\right) x_{0}-\beta_{0} x_{0}^{2}-\lambda x_{0}\left(y_{1}-\frac{a_{y}}{d_{2}} \eta_{2}\right)-\frac{\lambda d_{1}}{a_{x}} x_{1}^{2} \\
& -\gamma_{0}\left(y_{0}-\eta_{2}\right)^{2}-\lambda x_{1}\left(y_{0}-\eta_{2}\right)-\frac{\lambda \alpha d_{2}}{a_{y}} y_{1}^{2} \\
= & -T A T^{\prime}+\left(\beta-a_{x}-\lambda \frac{a_{y}}{d_{2}} \eta_{2}\right) x_{0} .
\end{aligned}
$$

The matrix $A$ is positive definite if $d_{1}>\frac{1}{4} \frac{\lambda a_{x}}{\gamma_{0}}$ and $d_{2}>\frac{1}{4 \alpha} \frac{\lambda a_{y}}{\beta_{0}}$. It follows from $q>\alpha$ and $d_{2} \leq d_{2}^{*}$ that $\dot{V}_{2} \leq 0$ for all $x_{0}, x_{1}, y_{0}, y_{1}$ and $\dot{V}_{2}=0$, when $x_{0}=x_{1}=0, y_{0}=\eta_{2}$ and $y_{1}=$ $\frac{a_{y}}{d_{2}} \eta_{2}$, hence La Salle's theorem [4] implies convergence of the solutions to this equilibrium, for all initial values not in the set $\mathbb{R}_{+}^{2} \times\{0\} \times \mathbb{R}_{+}$. This shows that the disease equilibrium $E_{2}$ is globally asymptotically stable in $\mathbb{R}_{+}^{4} / \mathbb{R}_{+}^{2} \times\{0\} \times \mathbb{R}_{+}$. If the initial data starts from $\mathbb{R}_{+}^{2} \times\{0\} \times \mathbb{R}_{+}$, then the solution obviously converges to the equilibria $E_{0}$ or $E_{1}$.

3. Let us consider the Lyapunov function

$$
\begin{aligned}
V_{3}= & \left(x_{0}-\xi_{3}-\xi_{3} \ln \frac{x_{0}}{\xi_{3}}\right)+\frac{\lambda}{2 a_{x}}\left(x_{1}-\frac{a_{x}}{d_{1}} \xi_{3}\right)^{2} \\
& +\left(y_{0}-\eta_{3}-\eta_{3} \ln \frac{y_{0}}{\eta_{3}}\right)+\frac{\lambda \alpha}{2 a_{y}}\left(y_{1}-\frac{a_{y}}{d_{2}} \eta_{3}\right)^{2} .
\end{aligned}
$$

It is easily seen that $V_{3} \geq 0$ and $V_{3}=0$ if and only if $x_{0}=\xi_{3}, x_{1}=\frac{a_{x}}{d_{1}} \xi_{3}, y_{0}=\eta_{3}$ and $y_{1}=\frac{a_{y}}{d_{2}} \eta_{3}$. Calculating the time derivative of $V_{3}$ along the positive solutions of model (10), we obtain

$$
\begin{aligned}
\dot{V}_{3}= & -\beta_{0}\left(x_{0}-\xi_{3}\right)^{2}-\lambda\left(x_{0}-\xi_{3}\right)\left(y_{1}-\frac{a_{y}}{d_{2}} \eta_{3}\right)-\frac{\lambda d_{1}}{a_{x}}\left(x_{1}-\frac{a_{x}}{d_{1}} \xi_{3}\right)^{2}-\gamma_{0}\left(y_{0}-\eta_{3}\right)^{2} \\
& -\lambda\left(x_{1}-\frac{a_{x}}{d_{1}} \xi_{3}\right)\left(y_{0}-\eta_{3}\right)-\frac{\lambda \alpha d_{2}}{a_{y}}\left(y_{1}-\frac{a_{y}}{d_{2}} \eta_{3}\right)^{2} \\
= & -T A T^{\prime} .
\end{aligned}
$$


The matrix $A$ is positive definite if $d_{1}>\frac{1}{4} \frac{\lambda a_{x}}{\gamma_{0}}$ and $d_{2}>\frac{1}{4 \alpha} \frac{\lambda a_{y}}{\beta_{0}}$. It follows from $d_{1}>d_{1}^{*}$ and $d_{2}>\max \left(d_{2}^{*}, d_{2}^{\circ}\right)$ that $\dot{V}_{3} \leq 0$ for all $x_{0}, x_{1}, y_{0}, y_{1}$ and $\dot{V}_{3}=0$ for $x_{0}=\xi_{3}, x_{1}=\frac{a_{x}}{d_{1}} \xi_{3}$, $y_{0}=\eta_{3}$ and $y_{1}=\frac{a_{y}}{d_{2}} \eta_{3}$. Hence La Salle's theorem [4] implies convergence of the solutions to this equilibrium, for all initial values not in the set $\{0\} \times \mathbb{R}_{+}^{3} \cup \mathbb{R}_{+}^{2} \times\{0\} \times \mathbb{R}_{+}$. This shows that the endemic equilibrium $E_{3}$ is globally asymptotically stable in $\mathbb{R}_{+}^{4} /\{0\} \times \mathbb{R}_{+}^{3} \cup \mathbb{R}_{+}^{2} \times\{0\} \times \mathbb{R}_{+}$. If the initial data starts from $\{0\} \times \mathbb{R}_{+}^{3} \cup \mathbb{R}_{+}^{2} \times\{0\} \times \mathbb{R}_{+}$, then the solution obviously converges to the equilibria $E_{0}, E_{1}$ or $E_{2}$.

\section{References}

[1] C. Castillo-Chavez and B. J. Song, Dynamical models of tuberculosis and their applications, Math. Biosci. Eng. 1 (2004) 361-404.

[2] P. Kim, P. Lee and D. Levy, Dynamics and potential impact of the immune response to chronic myelogenous leukemia, PLoS Comput. Biol., 4 (2008) 6, e1000095.

[3] N. L. Komarova and D. Wodarz, Drug resistance in cancer: Principles of emergence and prevention, Proc Natl Acad Sci USA, 102 (2005) 27, 9714-9719.

[4] J. P. LaSalle, The Stability of Dynamical Systems, CBMS-NSF Regional Conf. Ser. in Appl. Math. 25, SIAM, Philadelphia, 1976.

[5] F. Michor, T.P. Hughes, Y. Iwasa, S. Branford, N.P Shah, C.L. Sawyers and M. A. Nowak, Dynamic of chronic myeloid leukemia, Nature 435, 2005.

[6] I. Roeder, M. Horn, I. Glauche, A. Hochhaus, M. C. Mueller and M. Loeffler, Dynamic modeling of IMATINIB-treated chronic myeloid leukemia, functional insights and clinical implications, Nat. Med., 12 (2006) 10, 1181-1184. 